Supporting Information

\title{
Tetrahedral Framework Nucleic Acids Induce Immune Tolerance and Prevent the Onset of Type 1 Diabetes
}

Shaojingya Gao ${ }^{1 \ddagger}$, Yanjing $L^{1}{ }^{1}$, Dexuan Xiao ${ }^{1}$, Mi Zhou $^{1}$, Xiaoxiao Cai ${ }^{1}$, Yunfeng Lin ${ }^{1,2, *}$

${ }^{1}$ State Key Laboratory of Oral Diseases, West China Hospital of Stomatology, Sichuan University, Chengdu, Sichuan 610041, China

${ }^{2}$ College of Biomedical Engineering, Sichuan University, Chengdu, Sichuan 610041, China

\$ These authors contributed equally to this work.

* Corresponding author:

Yunfeng Lin

E-mail address: yunfenglin@scu.edu.cn 


\section{Materials and Methods}

Synthesis of TDN. The procedure of synthesizing tFNAs was described in previous studies (Table S1) ${ }^{1-2}$. The tFNAs composed of four prefabricated ssDNAs. The four ssDNAs were mixed in tri-base $\mathrm{MgCl}_{2}$ (TM) buffer, then the mixture heated at $95^{\circ} \mathrm{C}$ for 10 minutes for denaturation and immediately followed by $4^{\circ} \mathrm{C}$ for 20 minutes for pairing.

Characterization of tFNAs. To verify the successful fabrication of tFNAs, the measurements were taken similar to previous reports ${ }^{3-8} .8 \%$ PAGE and HPCE (Bioptic, Qsep100, Taiwan) were utilized to determine the theoretical molecular weights of ssDNA and combinations of two, three ssDNAs, and tFNAs. AFM (SPM-9700 Instrument; Shimadzu, Kyoto, Japan) and TEM (HITACHI HT7700, Tokyo, Japan) were employed to verify the morphology of tFNAs. Zeta potential and average size of tFNAs were examined by dynamic light scattering by Zetasizer Nano ZS90 (Malvern Instrument Ltd., Malvern, UK).

Mice. Female NOD/ShiLtJ mice were used as T1D animal model, while female ICR mice were served as normal mice. NOD and ICR mice were purchased from Jiangsu GemPharmatech Co, Ltd. Mice were housed in a specific pathogen-free animal facility, with unrestricted access to standard water and diet. Ethics committee of Sichuan University approved all animal procedures. Thirty-two female NOD mice aged nine weeks were treated with the $100 \mu \mathrm{L}$ of $250 \mathrm{nM}$ tFNAs, $100 \mu \mathrm{L}$ of $500 \mathrm{nM}$ tFNAs (dissolved in normal saline) or normal saline by tail-vein injection every other day for consecutively four weeks. Non-fasting blood glucose was monitored weekly for follow-up studies. Mice with two consecutive blood glucose levels exceeded $14 \mathrm{mmol} / \mathrm{L}$ were considered diabetic. Mice at 18 weeks, 24 weeks and 30 weeks of age were euthanized using Zoletil ${ }^{\circledR} 50$ (Virbic, France) $50 \mathrm{mg} / \mathrm{mL}(25 \mathrm{mg} / \mathrm{mL}$ tiletamine hydrochloride $+25 \mathrm{mg} / \mathrm{mL}$ zolazepam hydrochloride) followed by eyeball exsanguination. Blood, spleen, and pancreas were collected for the experiments described below.

In vivo bioluminescence imaging (BLI). Another ten NOD mice (female, ten weeks) were employed for the biodistribution experiments. To detect the biodistribution of the tFNAs, 
NOD mice were injected $100 \mu \mathrm{L}$ of Cy5.5-labeled tFNAs $(250 \mathrm{nM})$ via caudal vein, respectively. The mice were $60 \mathrm{~min}$ after injection, the mice were euthanized using Zoletil®50 (Virbic, France) $50 \mathrm{mg} / \mathrm{ml}(25 \mathrm{mg} / \mathrm{mL}$ tiletamine hydrochloride $+25 \mathrm{mg} / \mathrm{mL}$ zolazepam hydrochloride) and major organs, including thymus, pancreas, lung, heart, liver, spleen and kidney were collected. Images were acquired by using an in vivo fluorescence imaging system (Bio-Real Quick View 3000, Austria).

Histologic analysis. Pancreas were fixed in 4\% paraformaldehyde, paraffin embedded, and sections were stained with $\mathrm{H} \& \mathrm{E}$. The sections of pancreas islet were scored. Islet inflammation was graded on the basis of the previous system: grade 0, no indication of insulitis; grade 1, $<25 \%$ infiltration; grade 2, 25-50\% infiltration; and grade $3>50 \%$ infiltration ${ }^{9}$.

Immunohistochemistry. For immunohistochemistry, pancreas sections were prepared from paraffin-embedded tissues. Immunohistochemistry stains employed primary antibodies, including mouse anti-CD8, anti-CD4 (Servicebio, Wuhan, China). ImageJ (National Institutes of Health, Bethesda, MD, USA) software was used to analyse the immunohistochemistry images.

Immunofluorescence. For immunofluorescence, pancreas sections were prepared from frozen tissue and stained with anti-insulin, anti-glucagon, anti-Ki67, anti-Foxp3 antibodies and deoxynucleotidyl TUNEL (Servicebio, Wuhan, China). For $\beta$-cell death analysis in vivo, TUNEL and insulin immunostaining were performed as described ${ }^{10}$. For $\beta$-cell proliferation analysis, sections were stained for Ki67 (Servicebio, Wuhan, China) and insulin. Then, fluorophore-conjugated secondary antibodies employed (Servicebio, Wuhan, China). $\beta$-cells were also counted using ImageJ software (National Institutes of Health, Bethesda, MD, USA).

Enzyme-linked immunosorbent assay (Elisa). The serum levels of insulin, C-peptide, IFN- $\gamma$, IL-17, IL-10 and TGF- $\beta$ were quantified with commercial Elisa kits (Meimian Biology, Wuhan, China), following the manufacturer's instructions. 
Flow cytometry. Mouse splenocytes were prepared from pancreas, spleen and blood of normal mice and NOD mice. Cells were stained with the fluorochrome-labelled surface mAbs: CD4 APC-Cy7 (BD Bioscience) and CD25 PE-Cy7 (BD Bioscience), and incubated at $4^{\circ} \mathrm{C}$ for $30 \mathrm{~min}$. For intracellular protein staining, the cells were stimulated with phorbol 12-myristate 13-acetate (100 ng/mL; Sigma), ionomycin (1 mg/mL; Sigma) and brefeldin A $\left(1 \mathrm{mg} / \mathrm{mL}\right.$; Sigma) at $37^{\circ} \mathrm{C}$ for $6 \mathrm{~h}$. Cells were then fixed, permeabilized and washed using BD Cytofix/Cytoperm Kit. After that, intracellular proteins were stained with IFN- $\gamma 847$ (BD Bioscience), IL-4 PE (BD Bioscience), IL-17 PerCP-Cy5.5 (BD Bioscience), Foxp3 Alexa Fluor 488 (eBioscience), H-2K ${ }^{\mathrm{d}}$ NRP-V7 Tetramer-KYNKANVFL PE (MBL) at $4{ }^{\circ} \mathrm{C}$ for 40 min. Cells were resuspended in Phosphate Buffered Saline (PBS). Stained lymphocytes were analysed by Attune Nxt Flow Cytometer (Thermo Fisher, USA) with FlowJo software version 10 (BD Bioscience).

Phosflow $^{\text {TM }}$ analysis Phosflow analysis was employed to measure the activation of STAT family in lymphocytes and $\mathrm{CD} 4^{+} \mathrm{T}$ cells according to the protocol provided by $\mathrm{BD}$ company (BD Biosciences). According to the protocol, cells were stained with the surface antibodies CD4 V500 (BD Bioscience) and Phosflow ${ }^{\mathrm{TM}}$ antibodies, including p-STAT1 Alexa Fluor 647 (BD Bioscience), p-STAT3 Alexa Fluor 488 (BD Bioscience) and p-STAT5 PE (BD Bioscience). Phosflow ${ }^{\mathrm{TM}}$ Fix Buffer (BD Bioscience) and Phosflow ${ }^{\mathrm{TM}}$ Fix Buffer (BD Bioscience) were used in the experiment.

Statistical analysis. Graphpad Prism 8 (GraphPad Software Inc., San Diego, CA, USA) was performed to do the data analysis and generate the graphs. All results were presented as mean \pm SD. or as percentages. The Kaplan Meier survival analysis tests were applied to analyse the incidence of diabetes and survival. Statistical comparisons were made using one-way or two-way analysis of variance (ANOVA). A confidence level of 95\% was considered significant. For all tests, statistical significance of $\mathrm{p}$ value is shown as $*, \mathrm{P}<0.05, * *, \mathrm{P}<$ $0.01, * * *, \mathrm{P}<0.001$, or not significant $\mathrm{P}>0.05$. 

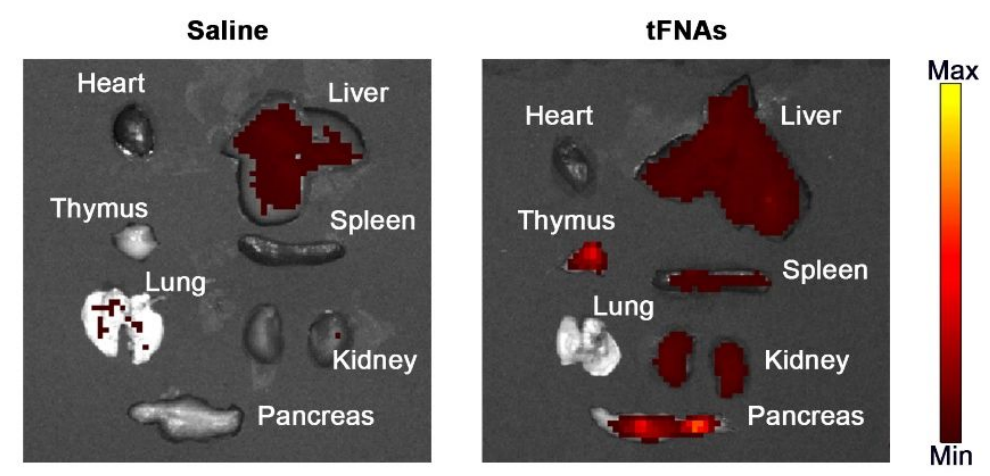

Figure S1. Fluorescence images of saline and tFNAs biodistribution in the major organs of NOD mice. 


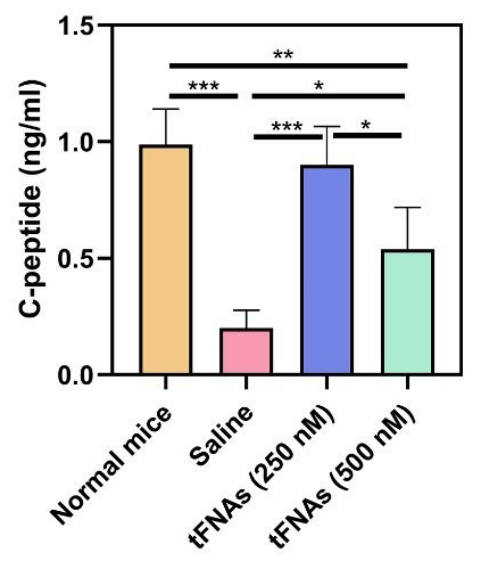

Figure S2. Blood c-peptide concentration. Blood c-peptide concentration in the 30-week-old mice of different groups (from three or four mice per group) as indicated. Throughout, data are expressed as means \pm SD. ${ }^{*} \mathrm{P}<0.05,{ }^{*} \mathrm{P}<0.01,{ }^{* * *} \mathrm{P}<0.001$. Statistical analyses were performed using one-way ANOVA. 

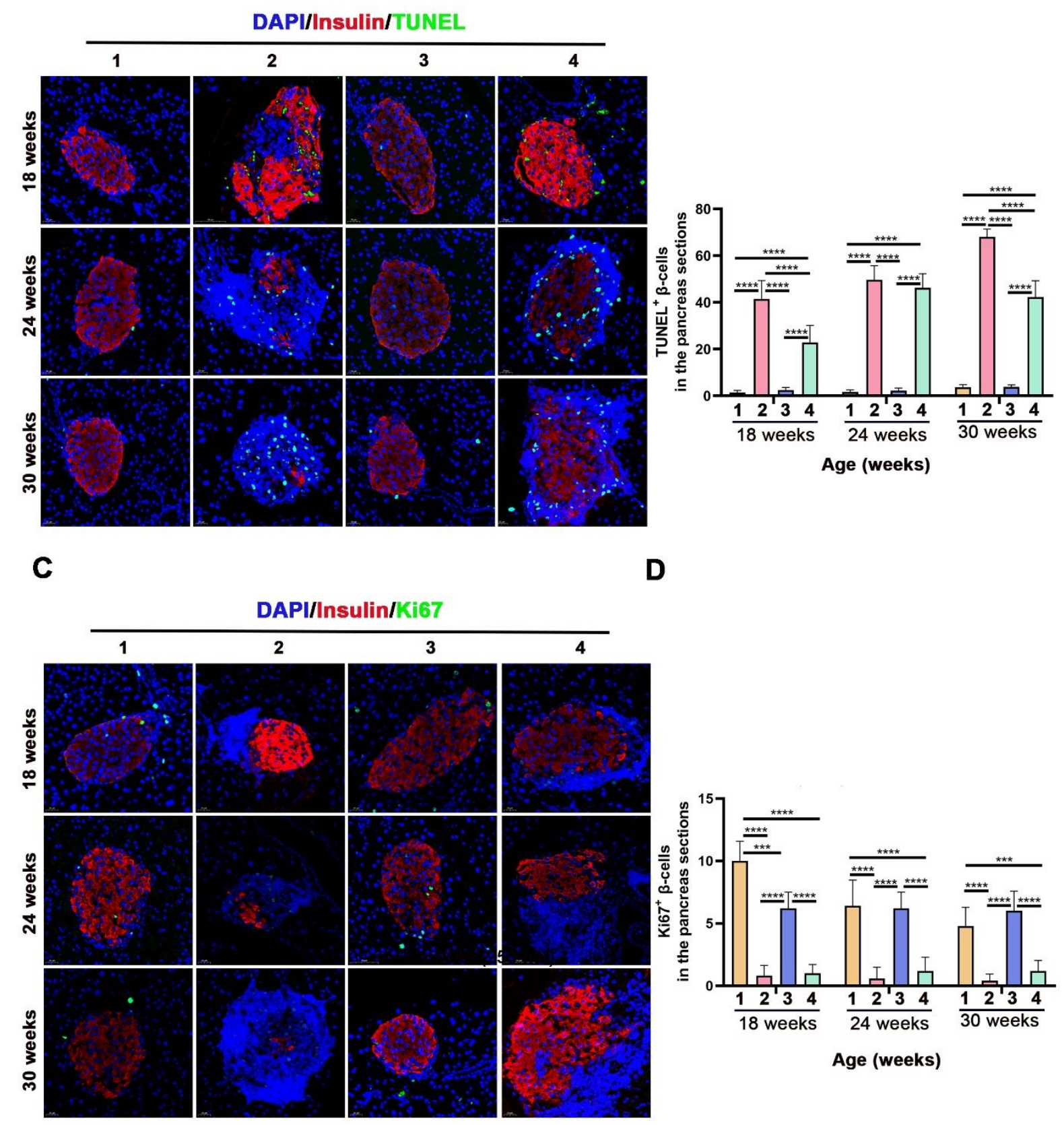

1, Normal mice; 2, Saline; 3, tFNAs (250 nM); 4, tFNAs (500 nM)

Figure S3. The tFNAs treatment preserved the mass of $\beta$-cells. A) Representative immunofluorescence images (from three or four mice per group) with the insets showing insulin-positive (red) and

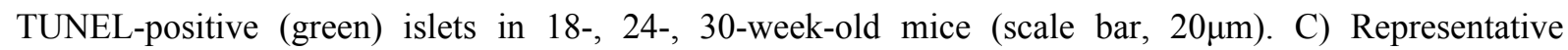
immunofluorescence images (from three or four mice per group) with the insets showing insulin-positive

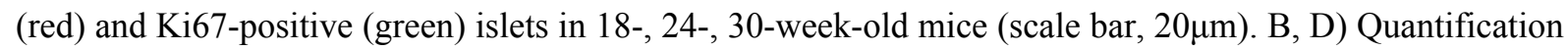
of $\mathrm{TUNEL}^{+}$and $\mathrm{Ki}^{+} 7^{+} \beta$-cells in the pancreas sections, respectively. Throughout, Data are expressed as 
means \pm SD. $* \mathrm{P}<0.05, * * \mathrm{P}<0.01, * * * \mathrm{P}<0.001$. Statistical analyses were performed using two-way ANOVA. 

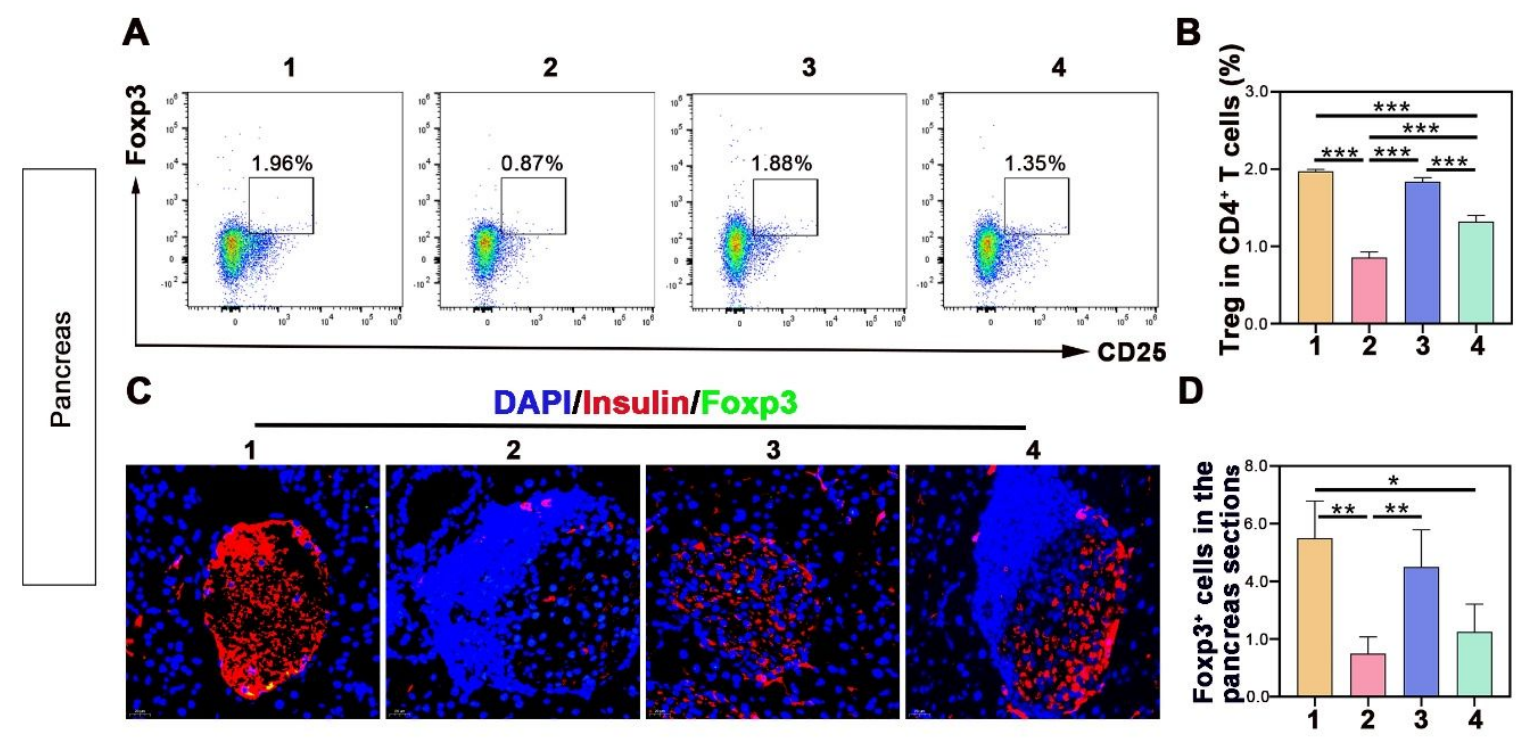

\section{1, Normal mice; 2, Saline; 3, tFNAs (250 nM); 4, tFNAs (500 nM)}

Figure S4. tFNAs affect pancreas-infiltrating Treg cells. (A) Representative dot blots (from four mice per group) of flow cytometry analyses for CD4+CD25+Foxp3+ T cells (Treg) obtained from the pancreas of 30-week-old mice. (B) Quantification of pancreas infiltrating Tregs. (C) Representative immunofluorescence images (from $t$ four mice per group) with the insets showing insulin-positive (red) and Foxp3-positive (green) islets in 30-week-old mice (scale bar, $20 \mu \mathrm{m}$ ). (D) Quantification of Foxp3+ cells in the pancreas sections. Throughout, data is presented by mean $\pm \mathrm{SD} .{ }^{*}, \mathrm{P}<0.05,{ }^{* *}, \mathrm{P}<0.01,{ }^{* * *}, \mathrm{P}<$ 0.001. Statistical analyses were performed using one-way ANOVA. 
A

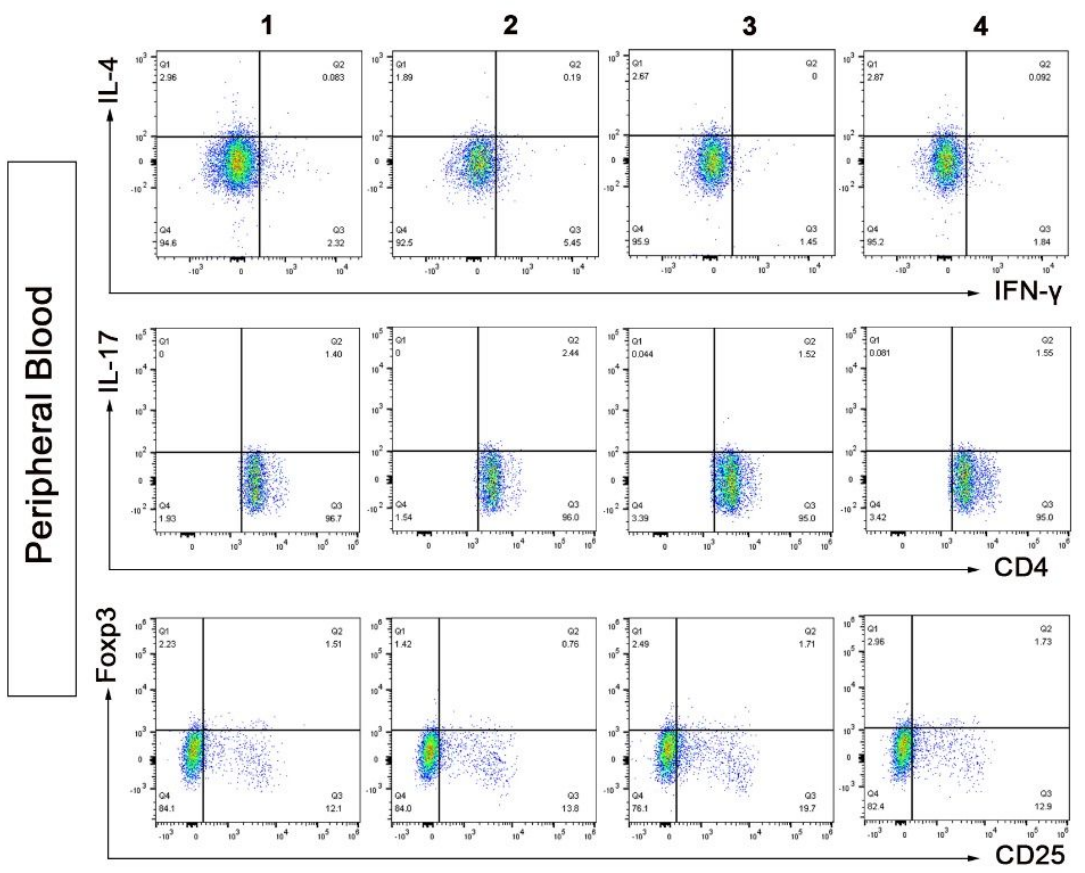

B
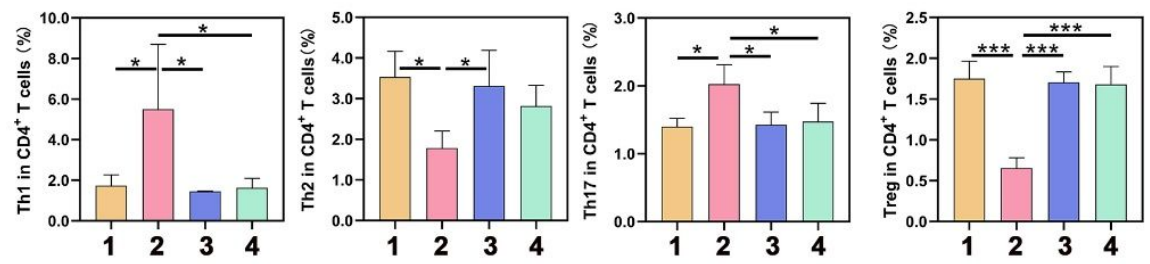

1, Normal mice; 2, Saline; 3, tFNAs (250 nM); 4, tFNAs (500 nM)

Figure S5. The tFNAs treatment regulated $\mathrm{CD}^{+} \mathrm{T}$ cells in peripheral blood of 18 -week-old mice. A) Representative plots of FACS analyses (from four or five mice per group) for $\mathrm{CD}^{+} \mathrm{IFN} \gamma^{+} \mathrm{T}$ cells (Th1 cells), $\mathrm{CD}^{+} \mathrm{IL}^{+} \mathrm{T}$ cells (Th2), $\mathrm{CD} 4^{+} \mathrm{IL} 17^{+} \mathrm{T}$ cells (Th17) and $\mathrm{CD} 4^{+} \mathrm{CD} 25^{+} \mathrm{Foxp} 3^{+} \mathrm{T}$ cells (Treg) obtained from the peripheral blood of 18-week-old mice, respectively. B) Percentage Th1, Th2, Th17 and Treg cells shown in $\mathrm{A}$. Throughout, data are expressed as means $\pm \mathrm{SD} .{ }^{*} \mathrm{P}<0.05,{ }^{*} \mathrm{P}<0.01,{ }^{* * *} \mathrm{P}<0.001$. Statistical analyses were performed using one-way ANOVA. 
A
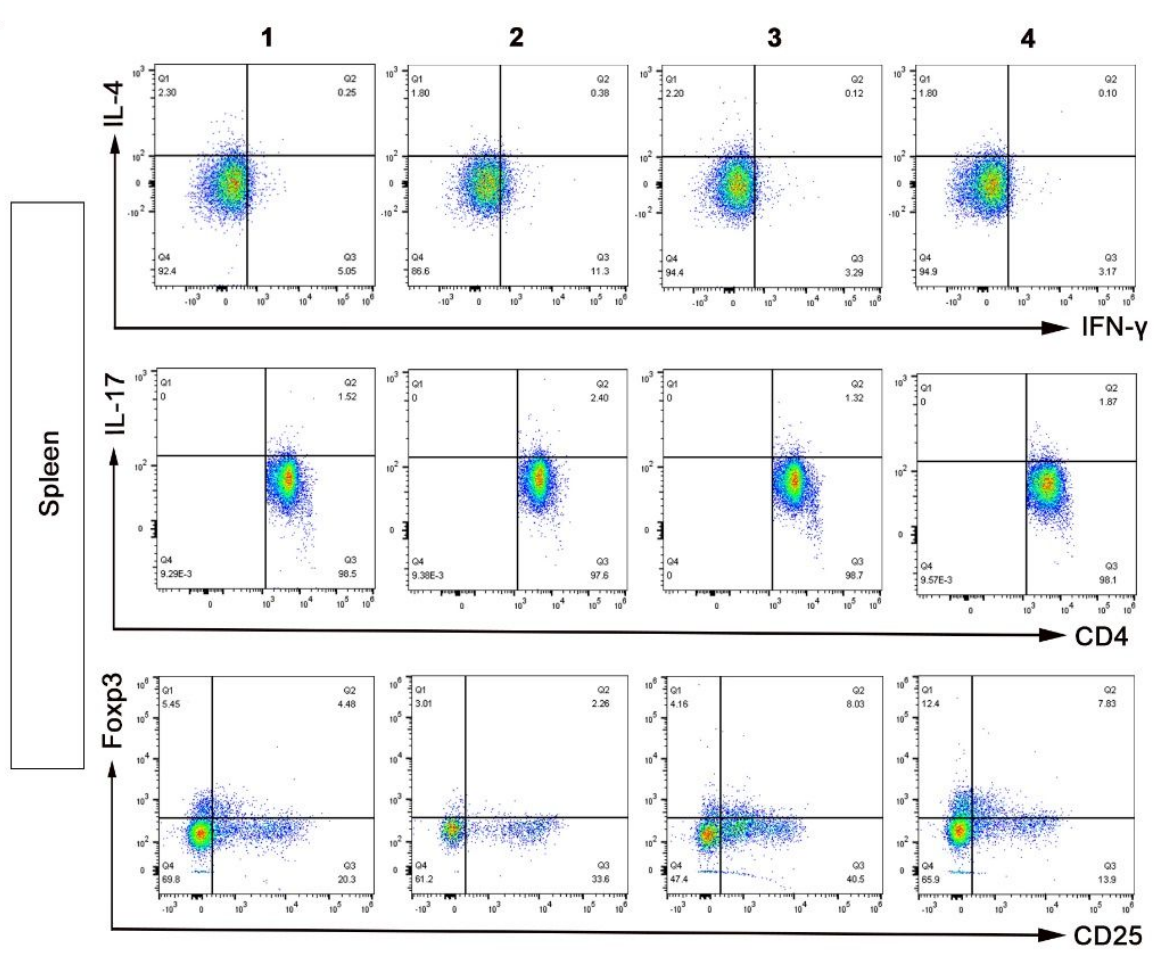

B
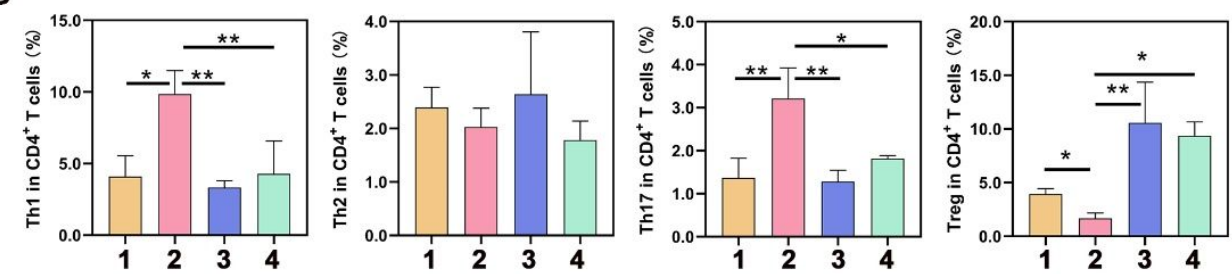

1, Normal mice; 2, Saline; 3, tFNAs (250 nM); 4, tFNAs (500 nM)

Figure S6. The tFNAs treatment regulated $\mathrm{CD}^{+} \mathrm{T}$ cells in spleen of 18 -week-old mice. A) Representative plots of FACS analyses (from four or five mice per group) for $\mathrm{CD} 4^{+} \mathrm{IFN} \gamma^{+} \mathrm{T}$ cells (Th1 cells), CD4 $4^{+} \mathrm{IL} 4^{+} \mathrm{T}$ cells (Th2), $\mathrm{CD} 4^{+} \mathrm{IL} 17^{+} \mathrm{T}$ cells (Th17) and $\mathrm{CD} 4^{+} \mathrm{CD} 25^{+} \mathrm{Foxp}^{+} \mathrm{T}$ cells (Treg) obtained from the spleen of 18-week-old mice, respectively. B) Percentage Th1, Th2, Th17 and Treg cells shown in A. Throughout, data are expressed as means $\pm \mathrm{SD} .{ }^{*} \mathrm{P}<0.05,{ }^{*} \mathrm{P}<0.01,{ }^{*} * \mathrm{P}<0.001$. Statistical analyses were performed using one-way ANOVA. 
A
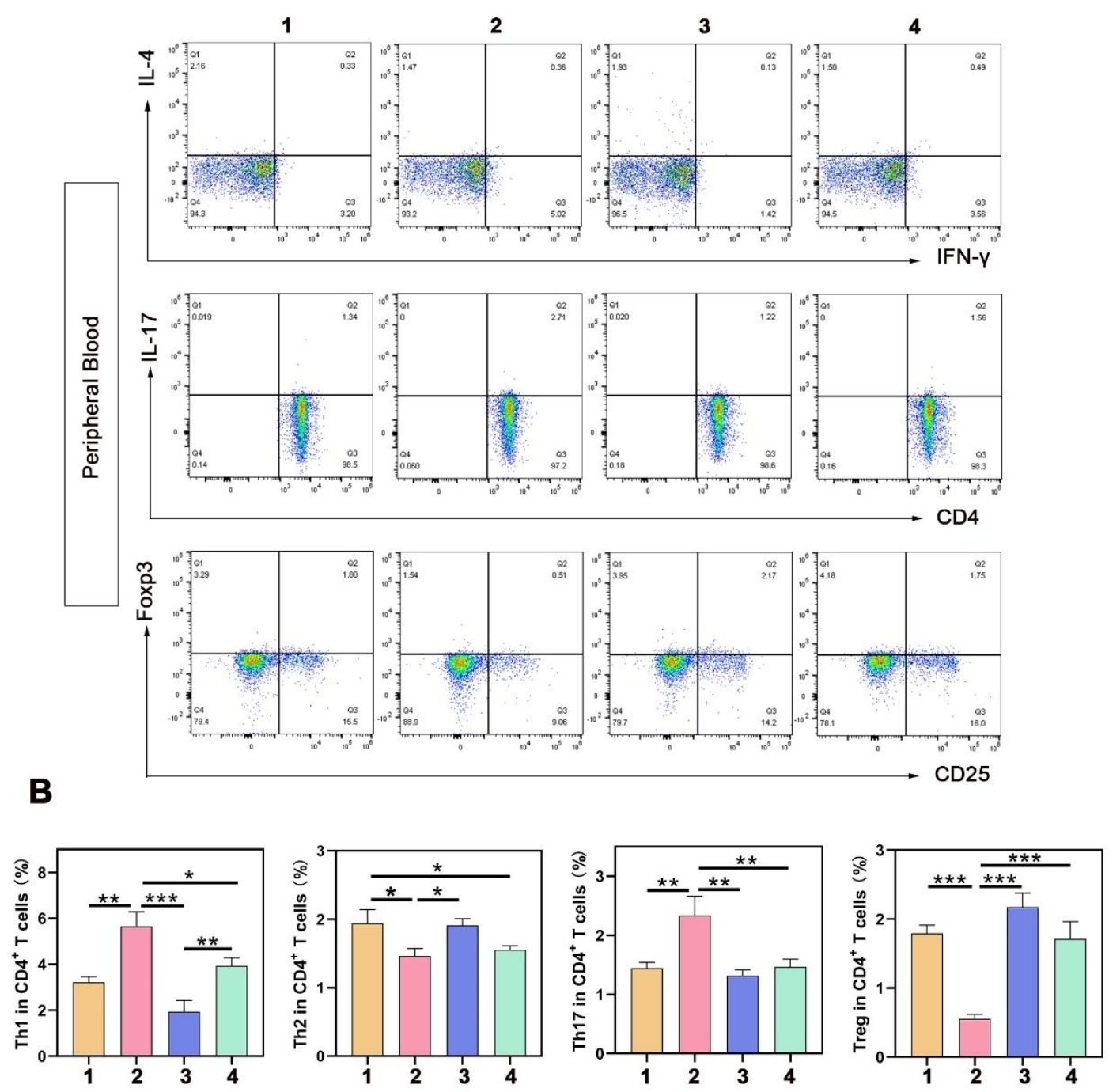

1, Normal mice; 2 , Saline; 3, tFNAs (250 nM); 4, tFNAs (500 nM)

Figure S7. The tFNAs treatment regulated $\mathrm{CD}^{+} \mathrm{T}$ cells in peripheral blood of 30 -week-old mice. A) Representative plots of FACS analyses (from four or five mice per group) for $\mathrm{CD}^{+} \mathrm{IFN} \gamma^{+} \mathrm{T}$ cells (Th1 cells), $\mathrm{CD} 4^{+} \mathrm{IL} 4^{+} \mathrm{T}$ cells (Th2), $\mathrm{CD} 4^{+} \mathrm{IL} 17^{+} \mathrm{T}$ cells (Th17) and $\mathrm{CD} 4^{+} \mathrm{CD} 25^{+} \mathrm{Foxp} 3^{+} \mathrm{T}$ cells (Treg) obtained from the peripheral blood of 30-week-old mice, respectively. B) Percentage Th1, Th2, Th17 and Treg cells shown in $\mathrm{A}$. Throughout, data are expressed as means $\pm \mathrm{SD} .{ }^{*} \mathrm{P}<0.05,{ }^{*} \mathrm{P}<0.01,{ }^{* * *} \mathrm{P}<0.001$. Statistical analyses were performed using one-way ANOVA. 
A

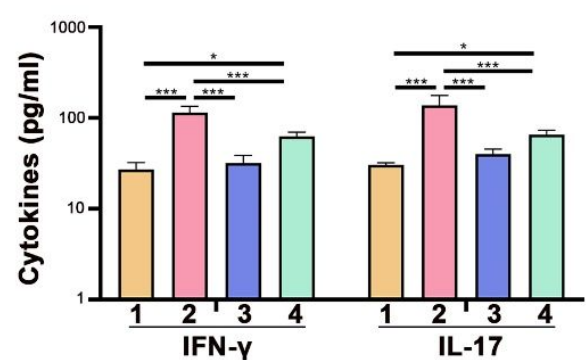

B

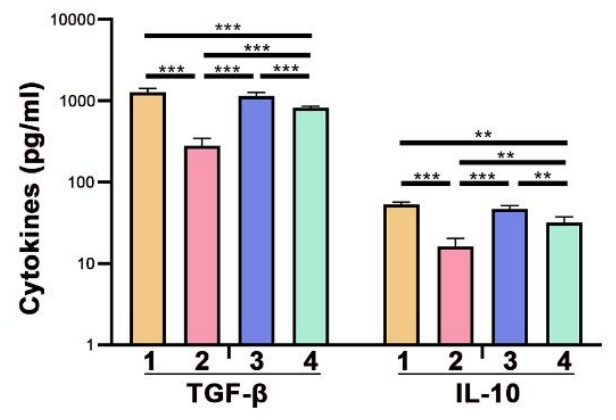

1, Normal mice; 2, Saline; 3, tFNAs (250 nM); 4, tFNAs (500 nM)

Figure S8. Concentration of cytokines in the blood. (A, B) Concentration of cytokines in the blood of the 30-week-old mice of different groups (from three or four mice per group) as indicated. Throughout, data are expressed as means $\pm \mathrm{SD} . * \mathrm{P}<0.05, * * \mathrm{P}<0.01, * * * \mathrm{P}<0.001$. Statistical analyses were performed using one-way ANOVA. 


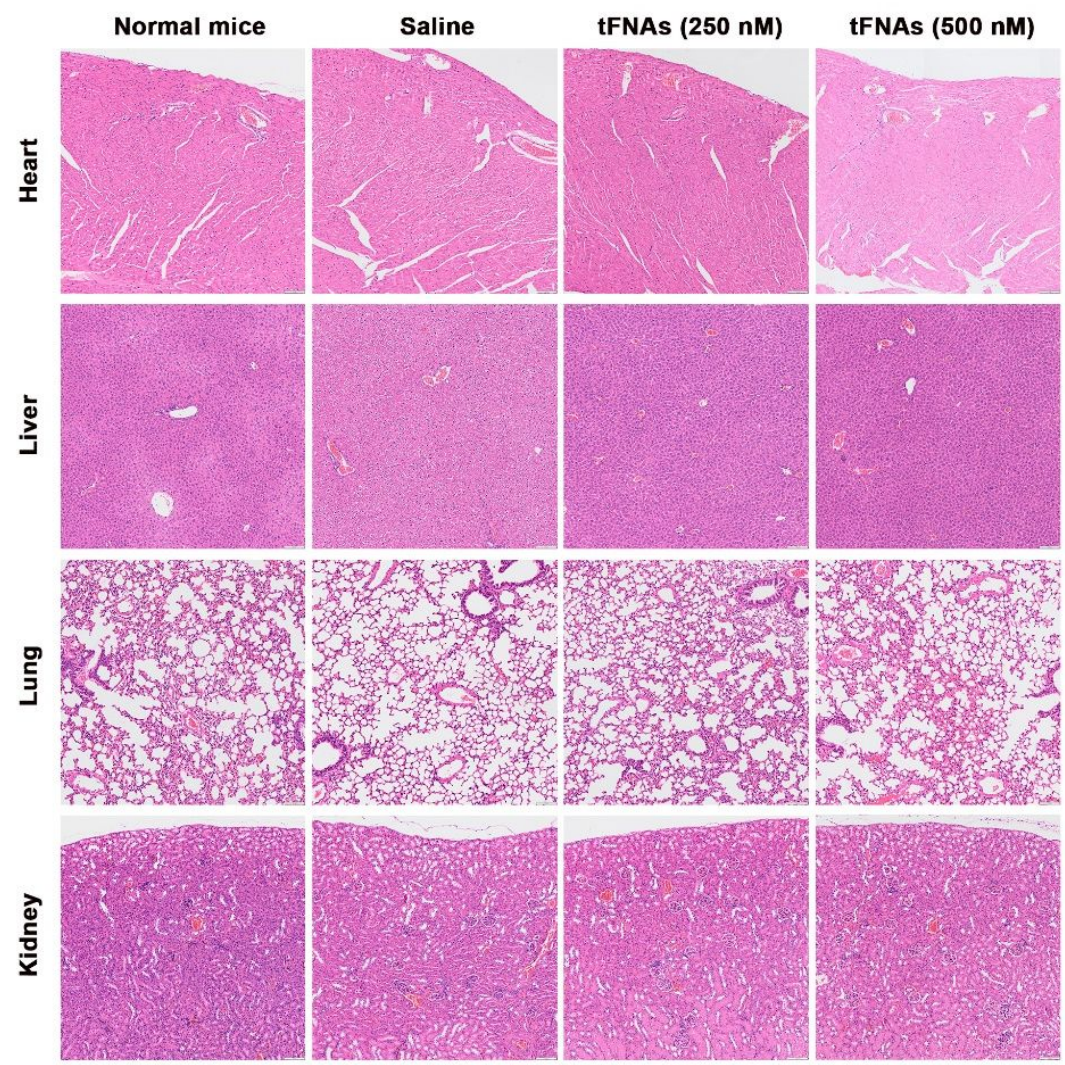

Figure S9. Histological assessment of the biocompatibility. No obvious toxicity was observed. 
Table S1 Base sequences of the ssDNAs used to construct the tFNAs.

ssDNA Base sequence $\left(5^{\prime} \rightarrow 3^{\prime}\right)$

S1 ATTTATCACCCGCCATAGTAGACGTATCACCAGGCAGTTGAGACGAACATTCCTAAGTCTGAA

S2 ACATGCGAGGGTCCAATACCGACGATTACAGCTTGCTACACGATTCAGACTTAGGAATGTTCG

S3 ACTACTATGGCGGGTGATAAAACGTGTAGCAAGCTGTAATCGACGGGAAGAGCATGCCCATCC

S4 ACGGTATTGGACCCTCGCATGACTCAACTGCCTGGTGATACGAGGATGGGCATGCTCTTCCCG 


\section{REFERENCES}

1. Li, S.; Tian, T.; Zhang, T.; Cai, X.; Lin, Y., Advances in biological applications of self-assembled DNA tetrahedral nanostructures. Materials Today 2019, 24, 57-68.

2. Zhang, T.; Tian, T.; Zhou, R.; Li, S.; Ma, W.; Zhang, Y.; Liu, N.; Shi, S.; Li, Q.; Xie, X.; Ge, Y.; Liu, M.; Zhang, Q.; Lin, S.; Cai, X.; Lin, Y., Design, fabrication and applications of tetrahedral DNA nanostructure-based multifunctional complexes in drug delivery and biomedical treatment. Nat Protoc 2020, 15 (8), 2728-2757.

3. Cui, W.; Zhan, Y.; Shao, X.; Fu, W.; Xiao, D.; Zhu, J.; Qin, X.; Zhang, T.; Zhang, M.; Zhou, Y.; Lin, Y., Neuroprotective and Neurotherapeutic Effects of Tetrahedral Framework Nucleic Acids on Parkinson's Disease in Vitro. ACS Appl Mater Interfaces 2019, 11 (36), 32787-32797.

4. Ge, Y.; Tian, T.; Shao, X.; Lin, S.; Zhang, T.; Lin, Y.; Cai, X., PEGylated Protamine-Based Adsorbing Improves the Biological Properties and Stability of Tetrahedral Framework Nucleic Acids. ACS Appl Mater Interfaces 2019, 11 (31), 27588-27597.

5. Liu, N.; Zhang, X.; Li, N.; Zhou, M.; Zhang, T.; Li, S.; Cai, X.; Ji, P.; Lin, Y., Tetrahedral Framework Nucleic Acids Promote Corneal Epithelial Wound Healing in Vitro and in Vivo. Small 2019, 15 (31), e1901907.

6. Qin, X.; Li, N.; Zhang, M.; Lin, S.; Zhu, J.; Xiao, D.; Cui, W.; Zhang, T.; Lin, Y.; Cai, X., Tetrahedral framework nucleic acids prevent retina ischemia-reperfusion injury from oxidative stress via activating the Akt/Nrf2 pathway. Nanoscale 2019, 11 (43), 20667-20675.

7. Zhu, J.; Zhang, M.; Gao, Y.; Qin, X.; Zhang, T.; Cui, W.; Mao, C.; Xiao, D.; Lin, Y., Tetrahedral framework nucleic acids promote scarless healing of cutaneous wounds via the AKT-signaling pathway. Signal Transduct Target Ther 2020, 5 (1), 120.

8. Zhou, M.; Liu, N. X.; Shi, S. R.; Li, Y.; Zhang, Q.; Ma, Q. Q.; Tian, T. R.; Ma, W. J.; Cai, X. X.; Lin, Y. F., Effect of tetrahedral DNA nanostructures on proliferation and osteo/odontogenic differentiation of dental pulp stem cells via activation of the notch signaling pathway. Nanomedicine 2018, 14 (4), 1227-1236.

9. Marino, E.; Richards, J. L.; McLeod, K. H.; Stanley, D.; Yap, Y. A.; Knight, J.; 
McKenzie, C.; Kranich, J.; Oliveira, A. C.; Rossello, F. J.; Krishnamurthy, B.; Nefzger, C. M.; Macia, L.; Thorburn, A.; Baxter, A. G.; Morahan, G.; Wong, L. H.; Polo, J. M.; Moore, R. J.; Lockett, T. J.; Clarke, J. M.; Topping, D. L.; Harrison, L. C.; Mackay, C. R., Gut microbial metabolites limit the frequency of autoimmune $\mathrm{T}$ cells and protect against type 1 diabetes. Nat Immunol 2017, 18 (5), 552-562.

10. Dirice, E.; Kahraman, S.; De Jesus, D. F.; El Ouaamari, A.; Basile, G.; Baker, R. L.; Yigit, B.; Piehowski, P. D.; Kim, M. J.; Dwyer, A. J.; Ng, R. W. S.; Schuster, C.; Vethe, H.; Martinov, T.; Ishikawa, Y.; Teo, A. K. K.; Smith, R. D.; Hu, J.; Haskins, K.; Serwold, T.; Qian, W. J.; Fife, B. T.; Kissler, S.; Kulkarni, R. N., Increased beta-cell proliferation before immune cell invasion prevents progression of type 1 diabetes. Nat Metab 2019, 1 (5), 509-518. 